\title{
Cross-Country Transportation Efficacy and Clinical Outcomes of Preloaded Large-Diameter Ultra-Thin Descemet Stripping Automated Endothelial Keratoplasty Grafts
}

\author{
Mohit Parekh, PhD, ${ }^{*} \dagger$ Alessandro Ruzza, BSc, ${ }^{*}$ Bernhard Steger, MD, $\ddagger$ Colin E. \\ Willoughby, MD,§ Salwah Rehman, MBChB, $\mid k$ Stefano Ferrari, PhD,* Diego Ponzin, MD,* \\ Stephen B. Kaye, MD, $\left\lceil\mathrm{k}\right.$ and Vito Romano, MD $\llbracket \mathrm{k}^{* *}$
} Purpose: To evaluate the clinical outcomes of preloaded large- diameter ultra-thin grafts for Descemet stripping
automated endo- thelial keratoplasty (UT-DSAEK) after cross-country shipment.

\begin{abstract}
Methods: A laboratory study in an eye bank and a clinical cohort study in an academic tertiary care center were performed. UT- DSAEK $(9.5 \mathrm{~mm}$ diameter) grafts $(\mathrm{n}=7)$ were prepared, loaded into a commercial device (iGlide; Eurobio, Les Ulis, France), preserved for 4 days at room temperature in transport medium, and analyzed. In a retrospective study, preloaded tissues $(n=39)$ for clinical use were prepared, transported from Italy to the United Kingdom, and surgically delivered into the eyes of patients undergoing UT- DSAEK. Central and peripheral endothelial cell density (ECD) and viability were measured before and after loading and storage of the grafts in the laboratory study. Clinically, best-corrected visual acuity, ECD before and at final follow-up, dislocation rate, primary graft failure, and surgical time were recorded.
\end{abstract}

Results: In the laboratory study, postcut central graft thickness was $93.3617 .2 \mathrm{~mm}$. ECD and cell mortality did not change significantly before and after preservation $(\mathrm{P}=0.8)$. Cell loss after 4 days of preservation was $1.7 \% 61.6 \%$. Clinically, 39 eyes of 39 patients at final follow-up showed a mean central graft thickness of $88622 \mathrm{~mm}$ and a bestcorrected visual acuity of 0.3460 .24 logMAR. Nine of 39 cases (23\%) needed rebubbling, and $28 \%$ cell loss was observed at final follow-up.

Conclusions: Large-diameter UT-DSAEK grafts can be prepared and preloaded in the eye bank using the iGlide and transported to the surgical center facilitating surgery for patients undergoing UT- DSAEK, potentially reducing tissue wastage, surgical time, and costs related to surgery.

Key Words: preloaded, corneal transplant, precut tissue, endothelial keratoplasty, UT-DSAEK

Introduction

Various endothelial keratoplasty (EK) techniques such as Descemet membrane EK (DMEK), Descemet stripping automated EK (DSAEK), and ultra-thin DSAEK (UT-DSAEK) have largely replaced penetrating keratoplasty for treatment of corneal disease resulting from endothelial failure. ${ }^{1-7}$ Neverthe- less, although there is a constant increment of DMEKs worldwide, DSAEK is still the most common EK performed because both its preparation and implantation methods are standardized. , $^{9}$

Endothelial graft survival is strongly dependent on donor endothelial cell density (ECD), ${ }^{10-12}$ which can be affected by donor selection, preparation, or surgical maneu- vers. Greenrod et al ${ }^{11}$ reported that after 2 years, graft survival in a large sample (1275 corneal transplants) was significantly lower if the donor selected had an ECD of less than 2400 cells $/ \mathrm{mm}^{2}$. We reported that larger EK grafts were signifi- cantly associated with a reduced failure rate. ${ }^{13}$ Furthermore, it has been reported that ECD increases from the center to the periphery of the cornea and in the peripheral zone; cells have a higher proliferative potential ${ }^{14}$ or so-called putative stem cells. In UT-DSAEK, graft preparation is a crucial step because graft thickness, endothelial integrity, and stromal surface smoothness determine the ultimate outcome. ${ }^{15-18}$ Production of a more standardized precut large-diameter UT-DSAEK graft may facilitate uptake of EK procedures in clinical practice with better postoperative visual outcomes. In our earlier report, we have shown that after preparation of an 8.5-mm graft for DSAEK, it can be preloaded in a glide (3D printed), transported, and implanted in a patient without complication. ${ }^{19}$ Preloaded tissues enable quality control in the eye bank, which is difficult to achieve in the surgical theater. We therefore evaluated both in vitro and 
in vivo, preparation, handling, and outcome of a 9.5-mm (large) UT-DSAEK graft preloaded in a commercially available iGlide device (Eurobio, Les Ulis, France).

\section{METHODS}

\section{Ethical Statement}

Human donor corneal tissues were used with written consent from the donor's next-of-kin to be used for research in the laboratory and for transplantation. Informed written consent was obtained from all the patients in the surgical setting. The Ethical Committee of Royal Liverpool University Hospital, Liverpool, United Kingdom, approved this retro- spective study. The study adhered to the principles of the Declaration of Helsinki.

\section{Preparation of Donor Tissues in the Eye Bank}

The preparation protocol has been previously described. ${ }^{17,19,20}$ Briefly, the corneas were mounted on an artificial anterior chamber (Moria, Antony, France) with appropriate intrachamber pressure. ${ }^{17,20}$ The corneas were allowed to reach a thickness between 500 and $510 \mathrm{~mm}$, measured using optical coherence tomography (Tomey, Nagoya, Japan). A microkeratome (Moria Evolution-3, Moria, Antony, France) with a $350-\mathrm{mm}$ depth blade was passed over the tissue with an aim of obtaining a posterior lamellar thickness of approximately $100 \mathrm{~mm}$ within the central $6 \mathrm{~mm}$. The peripheral anterior lamella of the donor corneal stroma from 9 to $11 \mathrm{~mm}$ was dissected using a $1.2-\mathrm{mm}$ crescent blade to ensure that a $9.5-\mathrm{mm}$ diameter punch only included the thinned tissue without any peripheral thickened edges. The endothelium was examined for mortal- ity and cell density. Soft silicone hydrogel contact lenses Clariti 1 day (Cooper Vision, Hamble, United Kingdom) were used as a base support to reduce any potential damage to the posterior lenticule during punching and the loading phase. The anterior cornea (both central and peripheral cornea that was dissected manually) was excised and replaced with the contact lens. Precut tissues were transferred to a standard punching block (Moria), with the endothelial side facing up. The tissues were trephined using a $9.5-\mathrm{mm}$ punch. The posterior lenticule was gently placed into the device along with the contact lens, filled with the transport medium, and preserved for 4 days at room temperature. Both, for laboratory and clinical studies, the tissues were gently flapped, with the endothelium inward inside the iGlide before releasing out for tissue analysis or implantation, respectively.

\section{Laboratory Study}

Human donor corneoscleral discs with ECD between 2000 and 2200 cells $/ \mathrm{mm}^{2}$ were used for the laboratory study. The tissues were preserved in tissue culture medium (TCM) at room temperature and then in deswelling solution/transport medium (TCM supplemented with 6\% dextran T500) before use. Age and sex of the cadaveric donors, postmortem time, time in TCM, and time in deswelling medium were recorded. All the tissues were stained using trypan blue $(0.25 \% \mathrm{wt} / \mathrm{vol})$ to evaluate the percentage of $\mathrm{dead} /$ necrotic cells and uncovered areas.

ECD and mortality (trypan blue-positive cells) were analyzed before preparation, after microkeratome cutting, and finally at the end of the preservation phase ( 4 days at room temperature). The endothelium was exposed to hypotonic sucrose solution to measure the number of endothelial cells and to examine the general morphology (pleomorphism and polymegathism). ECD was expressed as a mean of 5 different counts, each performed in triplicate at 5 different endothelial regions (one in the center and 4 in the periphery), at $\cdot 100$ magnification using a $1-\mathrm{mm}^{2} 10 \cdot 10$ reticule mounted in the eye piece of an inverted light microscope (Axiovert, Zeiss, Germany).

\section{Clinical Study}

Consecutive patients undergoing UT-DSAEK were included. The prepared lenticules were loaded into the iGlide (2.5 $\mathrm{mm}$ front opening), with the endothelial side facing the cap and the stromal side touching the contact lens to ensure no damage to the endothelial cells as described earlier. The cap was closed, and the iGlide was placed in the container with transport medium (Cornea Jet, Eurobio, Les Ulis, France) and shipped from the Venice Eye Bank, Italy, to the surgeons based in Liverpool, United Kingdom, at room temperature. The surgical procedure has been previously described. ${ }^{13,19}$ Briefly, in theater, complete 
descemetorhexis was performed. The glide arrived in the theater, with the cap on and the tissue resting on the contact lens (Fig. 1A). The cap of the glide was opened, and the preservation liquid was removed from the glide. The graft was separated from the contact lens, and viscoelastic solution was topically added on the endothelial side (Fig. 1B). The cap of the glide was closed and inverted to let the stromal side face the air. The graft was slightly pulled outside the $2.5-\mathrm{mm}$ front opening of the iGlide (Fig. 1C) and prepared for delivery. Using the pull-through technique, the precut- preloaded tissue was delivered using a 23-G forceps under the infusion of balanced salt solution through an anterior chamber maintainer in the recipient eye by gently pulling the tissue (Fig. 1D). The iGlide was not removed until the tissue was completely inside the eye. The tissue was not released until the graft was fully delivered, opened, and attached (Fig. 1E). Whenever necessary, the eye was gently tapped on the top to ensure that the graft opened up. After securing the tissue, the forceps was released, and using air as a tamponade, the tissue was attached to the recipient stroma, followed by suturing the eye, marking completion of surgery (Fig. 1F).

The main outcome measures were graft stability in the iGlide during transportation, graft dislocation, primary graft failure, and surgical time. Best spectacle-corrected visual acuity, ECD, corneal graft thickness after surgery, intra- operative and postoperative complications, adverse events, and adverse reactions were recorded. ECD was measured using a noncontact specular microscope (CellChekXL, Konan Medical, Hyogo, Japan). Graft thickness was measured using anterior segment optical coherence tomography (Tomey, Nagoya, Japan). Changes in ECD before and preparation, prior to following transplantation were analyzed using a Kruskal-Wallis test.

\section{RESULTS}

\section{Laboratory Study}

Human donor corneas $(n=7)$ from 5 men and 2 women with a mean (6SD) age of 56610 years were used. The time

from death to retrieval was 10.367 .7 hours, and the preservation (storage) time was 21.169 .4 days. Mortality, uncovered areas, and ECD at different times and regions are summarized in Supplemental Table 1 (see Supplemental Digital Content 1, http://links.lww.com/ICO/A718). There was no significant difference in ECD or cell mortality at different time points, that is, before preparation (Fig. 2A), after preparation (Fig. 2B), and after preservation of the preloaded graft (Fig. 2C) either in the center or periphery $(\mathrm{P}=0.8)$. Overall endothelial cell loss $(\mathrm{ECL})$ from preservation to use at 4 days was $1.7 \% 61.6 \%$ (average mortality of both scattered and the folds-if any). Although not significant, the reduction in ECD after preservation was slightly more apparent in the center than in the 4 peripheral zones, that is, center: 4.8\%; peripheral zones, P1: 2.0\%, P2: 1.3\%, P3: 1.4\%, and P4: 1.9\%. Postcut graft thickness was 93.36 $17.2 \mathrm{~mm}$.

\section{Clinical Study}

Thirty-nine eyes of 39 patients undergoing 9.5-mm UT- DSAEK preloaded in the iGlide were included, of which 13 were combined with cataract surgery. Thirty-nine tissues from 25 male and 14 female donors, with a mean age of 59.5613 .0 years, were used for this study. The postmortem time (from death to corneal harvest) was 11.965 .7 hours, and the tissues were preserved in TCM at $31^{\circ} \mathrm{C}$ for 11.464 .0 days before preloading. Mean central graft thickness was $83.5614 .5 \mathrm{~mm}$ (minimum-maximum: 42-105 mm) (Fig. 3A). ECD of the donor tissue was 26416178 cells $/ \mathrm{mm}^{2}$ and 2591699.6 cells $/ \mathrm{mm}^{2}$ before and after cutting, respectively, with an approximate cell loss of $1.9 \%$.

Mean central graft thickness was $98637 \mathrm{~mm}$ at initial follow-up and $88622 \mathrm{~mm}$ (minimum-maximum: 48- $134 \mathrm{~mm}$ ) (Figs. 3B, C) at final follow-up (8.5 months). In 9/39 cases (23\%), rebubbling during the first week after surgery was required with successful graft reattachment. In vivo ECD was 18636167 cells $/ \mathrm{mm}^{2}$ at final follow-up, with an average of $28.1 \%$ cell loss compared with that measured in the eye bank before releasing the tissue, which did not differ significantly from the data previously published by Ruzza et $\mathrm{al}^{19}$ (25\% cell loss at 6 months). Best-corrected visual acuity at final follow-up was 0.3460 .24 logMAR. No adverse events or reactions have been recorded when precut DSAEK tissues have been internationally shipped ${ }^{21}$; simi- larly, we did not record any negative events during graft preparation at the eye bank, during transportation of the tissue, intraoperatively, or postoperatively for precut, pre- 
loaded large-diameter UT-DSAEK grafts that were interna- tionally shipped. The overall time required for surgery never exceeded 20 minutes (from opening of the vial until suturing).

\section{DISCUSSION}

DSAEK has become a reproducible EK technique with currently a lower complication rate than DMEK.22 UT- DSAEK has been shown to achieve better best spectacle- corrected visual acuity and faster visual recovery than DSAEK. ${ }^{23}$ Busin et al ${ }^{2}$ also reported that UT-DSAEK (graft

Copyright (C) 2018 Wolters Kluwer Health, Inc. All rights reserved.

thickness, $100 \mathrm{~mm}$ ) is able to achieve best-corrected visual acuity close to results reported after DMEK.

Preloaded donor tissue helps to reduce the surgical time and some of the risks associated with preparation of large- diameter UT-DSAEK grafts in the operating room. Eye banks receive and process tissue and meet requests in continually changing scenarios, such as, for example, prestripped, precut, or preloaded tissue for DSAEK and DMEK. ${ }^{23,24}$ Preparation of the tissue by the eye bank benefits the eye bank, surgeon, and, importantly, the patient. It may lead to reduced tissue wastage, preparation errors, quality-assured grafts, and a rel- ative reduction in overall costs due to continuous usage of instruments and facilities in the eye bank. It enables the surgeons to obtain preloaded or preprepared tissue, thereby facilitating surgery and reducing the surgical time and costs, which help outweigh the cost of preloading the tissue in the eye bank.

Clinically, we did not encounter any graft dislocation events during the transportation phase. This is attributable to the cylindrical shape of the delivery device, which maximizes adherence of the contact lens that supports the corneal stroma to the inner wall of the device, and a glide cap, which prevents the tissue from floating out into the fluid in the transport bottle. In our previous study ${ }^{19}$ we used the anterior lenticule as a support for the posterior graft inside the iGlide; however, it was observed that the posterior lenticule attaches to the anterior lenticule strongly when preserved for more than 24 hours and creates difficulties while excising the posterior lenticule during implantation. Using the contact lens offered advantages such as holding the tissue inside the iGlide without a lot of movement and ease of releasing the graft from the contact lens for transplantation.

Although it was not a comparative series, this is the largest case series of preloaded large UT-DSAEK reported so far. The absence of intraoperative complications would suggest that the iGlide is easy to handle, facilitating delivery of the tissue into the eye. Although there was a slight increase in detachments to $23 \%$, they were easily rescued using air bubbles. A possible explanation for this increase may reflect movement of storage medium over the graft during shipping, leading to a change and smoothing of the stromal surface. As seen earlier, if present, this loss of roughening may reduce adhesion. ${ }^{25}$

Although a larger graft diameter is associated with a decreased distance between the graft edge and the limbal vessels, we have previously shown that graft survival is in fact improved in larger-diameter UTDSAEK ${ }^{13}$ It is also noteworthy that the reduction in ECD between that measured in vitro in the laboratory and subsequently measured in vivo after surgery did not differ as much as previously reported. ${ }^{26}$ With minimal endothelial cell damage during preparation, transportation, and transplantation, the iGlide serves as a feasible carrier to transport the cells from an eye bank to the patient. In the earlier published article, ${ }^{19}$ we also investigated cell apoptosis and tight junction proteins and noted that the tissues when preloaded did not show any significant apoptosis but presented expression of ZO-1 (zonula occludens-1, tight junction protein). Although spec- ulative, because the tissues were preserved in the same conditions and with a lower time of preservation, it would be reasonable to expect that larger-diameter grafts would also not show any apoptosis and would similarly express ZO-1.

In agreement with previous results, ${ }^{19}$ storage of large precut grafts inside the iGlide did not appear to change endothelial organization and was not associated with increased peripheral endothelial cell loss. This study confirms application of the protocol used for UT graft preparation to larger-diameter grafts to achieve a graft thickness of $100 \mathrm{~mm}$ centrally and 4 days of preservation. Although we have reported that preload- ing DSAEK grafts did not lead to significant endothelial damage when preserved up to 7 days, ${ }^{19}$ we have observed that most DSAEK procedures are undertaken within 3-4 days after transportation of the tissue from the eye bank to the transplant center. This also provides a longer time frame for cross- 
country shipment as reported in this article. There is, however, no difference in endothelial damage when preserved up to 4 or 7 days. The results of this study provide additional evidence to support the proposed method to prepare and transport large- diameter UT grafts preloaded in an iGlide, shipped cross- country for facilitating DSAEK.

\section{REFERENCES}

1. Melles GR, Eggink FA, Lander F, et al. A surgical technique for posterior lamellar keratoplasty. Cornea. 1998;17:618-626.

2. Busin M, Madi S, Santorum P, et al. Ultrathin descemet's stripping automated endothelial keratoplasty with the microkeratome double-pass technique: two-year outcomes. Ophthalmology. 2013;120:1186-1194.

3. Terry MA, Straiko MD, Goshe JM, et al. Descemet's stripping automated endothelial keratoplasty: the tenuous relationship between donor thickness and postoperative vision. Ophthalmology. 2012;119:1988-1996.

4. Price FW, Price MO. Descemet's stripping with endothelial keratoplasty in 50 eyes: a refractive neutral corneal transplant J Refract Surg. 1995; 21:339-345.

5. Melles GRJ. Posterior lamellar keratoplasty: DLEK to DSEK to DMEK. Cornea. 2006;25:879-881.

6. Busin M, Leon P, Scorcia V, et al. Contact lens-assisted pull-through technique for delivery of tri-folded (endothelium in) DMEK grafts minimizes surgical time and cell loss. Ophthalmology. 2016;123:476-483.

7. Parekh M, Ruzza A, Ferrari S, et al. Endothelium-in versus endothelium- out for Descemet membrane endothelial keratoplasty graft preparation and implantation. Acta Ophthalmol (Copenh). 2017;95:194-198.

8. 8. Guerra FP, Anshu A, Price MO, et al. Endothelial keratoplasty: fellow eyes comparison of Descemet stripping automated endothelial kerato- plasty and Descemet membrane endothelial keratoplasty. Cornea. 2011; 30:1382-1386.

9. 9. Arnalich-Montiel F, Hernández-Verdejo JL, Oblanca N, et al. Compar-ison of corneal haze and visual outcome in primary DSAEK versus DSAEK following failed DMEK. Graefes Arch Clin Exp Ophthalmol. 2013;251:2575-2584.

10. 10. Wakefield MJ, Armitage WJ, Jones MNA, et al. The impact of donor age and endothelial cell density on graft survival following penetrating keratoplasty. Br J Ophthalmol. 2016;100:986-989.

11. 11. Greenrod EB, Jones MNA, Kaye S, et al. Center and surgeon effect on outcomes of endothelial keratoplasty versus penetrating keratoplasty in the United Kingdom. Am J Ophthalmol. 2014;158:957-966.

12. 12. Ishii N, Yamaguchi T, Yazu H, et al. Factors associated with graft survival and endothelial cell density after Descemet's stripping auto- mated endothelial keratoplasty. Sci Rep. 2016;6:25276.

13. 13. Romano V, Tey A, Hill NME, et al. Influence of graft size on graft survival following Descemet stripping automated endothelial kerato- plasty. Br J Ophthalmol. 2015;99:784-788.

14. 14. Yu WY, Sheridan C, Grierson I, et al. Progenitors for the corneal endothelium and trabecular meshwork: a potential source for personal-ized stem cell therapy in corneal endothelial diseases and glaucoma. J Biomed Biotechnol. 2011;2011:412743.

15. 15. Bucher F, Roters S, Mellein A, et al. "OSMO-UT-DSAEK” using THIN- C medium. Graefes Arch Clin Exp Ophthalmol. 2013;251:2181-2185.

16. 16. Thomas PBM, Mukherjee AN, O’Donovan D, et al. Preconditioned donor corneal thickness for microthin endothelial keratoplasty. Cornea. 2013;32:e173-178.

17. 17. Romano V, Steger B, Chen JY, et al. Reliability of the effect of artificial anterior chamber pressure and corneal drying on corneal graft thickness. Cornea. 2015;34:866-869.

18. 18. Busin M, Patel AK, Scorcia V, et al. Microkeratome-assisted preparation of ultrathin grafts for Descemet stripping automated endothelial kerato- plasty. Invest Ophthalmol Vis Sci. 2012;53:521-524.

19. 19. Ruzza A, Parekh M, Ferrari S, et al. Preloaded donor corneal lenticules in a new validated 3D printed smart storage glide for Descemet stripping automated endothelial keratoplasty. Br J Ophthalmol. 2015;99:1388- 1395.

20. 20. Romano V, Steger B, Myneni J, et al. Preparation of ultrathin grafts for Descemet stripping endothelial keratoplasty with a single microkeratome pass. J Cataract Refract Surg. 2017;43:12-15.

21. 21. Nakagawa H, Inatomi T, Hieda O, et al. Clinical outcomes in Descemet stripping automated endothelial keratoplasty with internationally shipped precut donor corneas. Am J Ophthalmol. 2014;157:50-55.e1.

22. 22. Phillips PM, Phillips LJ, Muthappan V, et al. Experienced DSAEK surgeon's transition to DMEK: outcomes comparing the last 100 DSAEK surgeries with the first 100 DMEK surgeries exclusively using previously published techniques. Cornea. 2017;36:275-279.

23. 23. Parekh M, Baruzzo M, Favaro E, et al. Standardizing descemet membrane endothelial keratoplasty graft preparation method in the eye bank - experience of 527 descemet membrane endothelial keratoplasty tissues. Cornea. 2017;36:14581466.

24. Parek M, M, Ruzza A, Ferrari S, et al. Preloaded tissues for descemet membrane endothelial keratoplasty. Am J Ophthalmol. 2016;166:120- 125

25. Bhogal MS, Angunawela RI, Bilotti E, et al. Theoretical, experimental, and optical coherence tomography (OCT) studies of graft apposition and adhesion in Descemets stripping automated endothelial keratoplasty (DSAEK). Invest Ophthalmol Vis Sci. 2012;53:3839-3846.

26. 26. Dickman MM, Kruit PJ, Remeijer L, et al. A randomized multicenter clinical trial of ultrathin descemet stripping automated endothelial keratoplasty (DSAEK) versus DSAEK. Ophthalmology. 2016;123: 2276-2284. 


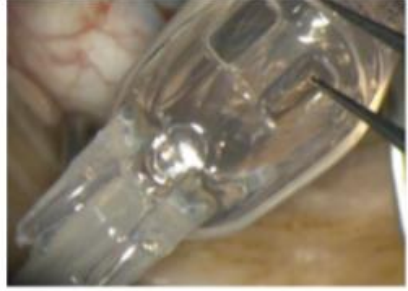

A

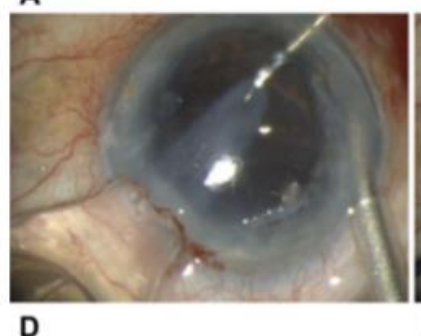

D

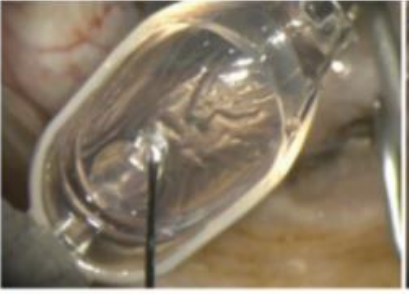

B

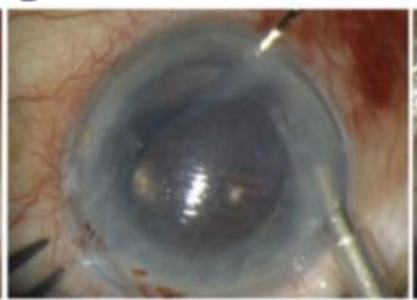

E
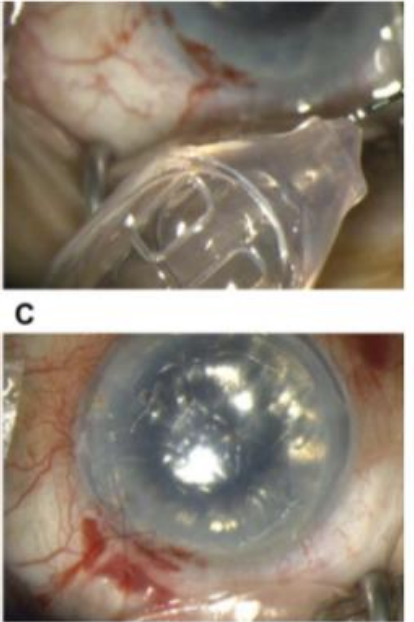

$\mathbf{F}$

FIGURE 1. Clinical approach for preloaded UT-DSAEK grafts in the iGlide. A, iGlide, as it arrives in the surgical theater. B, The cap of the glide was opened, and after removing all the liquid present in the glide, viscoelastic solution was topically applied on endothelial cells. C, The cap was closed, and the preloaded UT-DSAEK graft was gently pulled out of the glide pore slightly, using a forceps. D, With pull-through technique, the graft was inserted in the patient's eye. $E$, The glide was not removed until the graft was completely delivered. F, Once the tissue was completely inserted and attached, the eye was sutured, and surgery was completed.
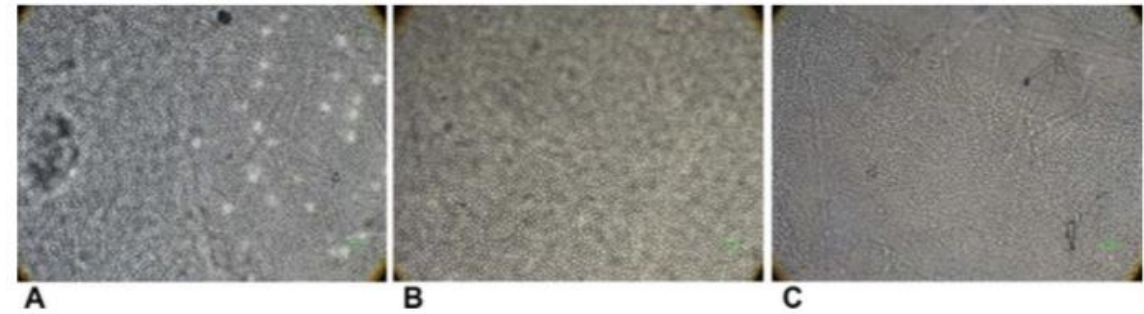

FIGURE 2. Endothelial cells evaluated using an inverted light microscope. A, Endothelial cells showing minimal to no mortality before cutting. B, The endothelial cells showed maintenance of morphology without mortality after cutting and (C) after preserving the tissues in the iGlide for 4 days at room temperature.

FIGURE 3. Anterior segment optical coherence tomography of donor tissue, (A) after cutting showing central and peripheral thickness, (B, C) after the delivery of the graft inside the patient's eye at final follow-up.
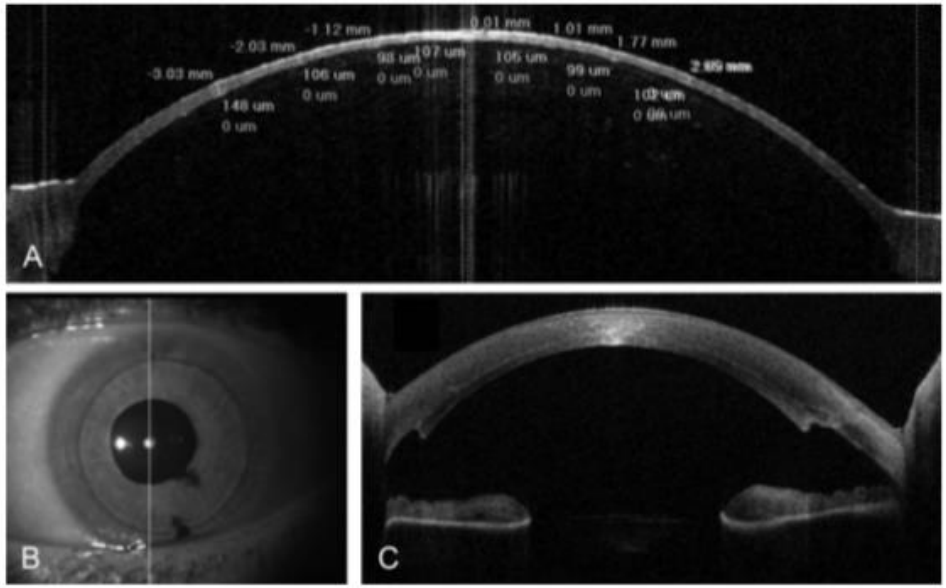